\title{
The Influence of a Community Pharmacy Automatic Prescription Refill Program on Medicare Part D Adherence Metrics
}

\author{
Corey A. Lester, PharmD, MS; David A. Mott, PhD; and Michelle A. Chui, PharmD, PhD
}

\begin{abstract}
BACKGROUND: The Centers for Medicare \& Medicaid Services (CMS) include measures of medication adherence within its Medicare Part D star ratings program. These adherence measures have motivated the development of new methods to improve patient adherence. Automatic prescription refill programs in community pharmacies are an intervention that has seen widespread adoption in recent years. These automatic refill programs anticipate and initiate prescription refills on a standardized, recurrent basis. As a result, prescription refills may be filled before a patient typically might initiate a refill. This study measures the effect of an automatic prescription refill program on 3 adherence metrics used by CMS within Medicare Part $D$ star ratings.
\end{abstract}

OBJECTIVE: To compare the value of Medicare Part $D$ adherence metrics for an automatic prescription refill program relative to standard prescription refills.

METHODS: Prescription dispensing data (January 1, 2014-December 31, 2014) from a chain of 29 pharmacies in a midwestern state were used to conduct this analysis. A post-only, quasi-experimental design separated patients into automatic and standard prescription refill cohorts. Refill adherence was calculated using proportion of days covered (PDC) for each of the 3 adherence metrics used by CMS for statins, renin angiotensin aldosterone system antagonists (RASA), and noninsulin diabetes medications. The adherence rate was defined as the proportion of patients with a PDC $\geq 80 \%$. Inclusion criteria for patients followed the Pharmacy Quality Alliance technical specifications. Chi-square analysis and multiple logistic regression were used to examine differences in $\mathrm{PDC} \geq 80 \%$ between the 2 study cohorts.

RESULTS: There were $1,018,1,006$, and 368 patients for the automatic refill cohort and 3,928, 3,409, and 1,207 patients for the standard refill cohort in the statin, RASA, and diabetes adherence metrics, respectively. The mean age [SD] of patients was between $79.2[ \pm 8.5]$ and $80.8[ \pm 9.9]$ years across all cohorts. Patients in the automatic prescription refill program tended to take less than 1 additional chronic medication compared with the standard refill prescription cohort. The proportion of adherent patients ranged from $73.6 \%$ to $76.4 \%$ for standard refill cohorts and $77.5 \%$ to $83.6 \%$ for automatic refill cohorts. Differences between study cohorts were statistically significant for all the adherence metrics based on the chi-square test $(P<0.05)$. Patients enrolled in the automatic prescription refill program were more likely to be adherent to the statin $(\mathrm{OR}=1.51,95 \% \mathrm{Cl}=1.26$ 1.82), RASA (OR=1.20, $95 \% \mathrm{Cl}=1.01-1.42)$, and diabetes $(0 \mathrm{R}=1.44,95 \%$ $\mathrm{Cl}=1.06-1.96)$ metrics.

CONCLUSIONS: Patients enrolled in the automatic prescription refill program were more likely to be adherent to their medications. Enrollment in automatic prescription refill programs could be encouraged by health plans and pharmacists because of their potential effect on Medicare Part $D$ star ratings.

J Manag Care Spec Pharm. 2016;22(7):801-07

Copyright $\odot 2016$, Academy of Managed Care Pharmacy. All rights reserved.

\section{What is already known about this subject}

The Centers for Medicare \& Medicaid Services (CMS) quality metrics to evaluate Medicare Advantage and Prescription Drug Plans are playing an increasingly important role. A major component of these metrics are those related to medication adherence. Automatic prescription refill programs have become commonplace in the community pharmacy industry over the last several years.

\section{What this study adds}

This study evaluates the effect of an automatic prescription refill program in community pharmacy on medication adherence metrics used by CMS.

The automatic prescription refill program that was evaluated resulted in a significant increase in the proportion of patients that were adherent to their medications for each of the 3 adherence metrics.

T The Pharmacy Quality Alliance (PQA) was established in 2006 to develop and endorse quality metrics that could be used to evaluate the quality of patient medication use and pharmacist patient care. ${ }^{1}$ To date, PQA has endorsed 30 metrics, including those that measure adherence to medication. Some of these metrics have been adopted by health care organizations, including the Centers for Medicare $\&$ Medicaid Services (CMS).

The CMS Medicare star rating program is used to assess quality of care throughout the health care industry. CMS implemented the program for Medicare Advantage and Prescription Drug Plans in 2009 to evaluate the quality of insurance plans on different aspects of care, including patient safety. CMS reports a star rating for each of the metrics and all contribute to calculating an overall star rating for each plan. There are 3 medication-related adherence metrics used by Medicare Part D that apply to statins, renin angiotensin aldosterone system antagonists (RASA), and noninsulin diabetes medications. These 3 metrics are triple weighted, which means they are valued more highly compared with other medication metrics in calculating star ratings for prescription drug plans. The 2016 adherence metrics, using 2014 contract data, account for $10.9 \%$ and $29.5 \%$ of the overall star rating calculation for Medicare Advantage and stand-alone prescription drug plans, respectively. ${ }^{2}$ 
The Influence of a Community Pharmacy Automatic Prescription Refill Program on Medicare Part D Adherence Metrics

TABLE 1 Patient Demographics and Adherence Results for Each Adherence Metric by Refill Program Type

\begin{tabular}{|c|c|c|c|c|c|c|}
\hline & \multicolumn{2}{|c|}{ Statin } & \multicolumn{2}{|c|}{ RASA } & \multicolumn{2}{|c|}{ Diabetes } \\
\hline & Automatic Refill & Standard Refill & Automatic Refill & Standard Refill & Automatic Refill & Standard Refill \\
\hline Number & 1,018 & 3,928 & 1,006 & 3,409 & 368 & 1,207 \\
\hline \multicolumn{7}{|l|}{ Gender, n (\%) } \\
\hline Male & $526 \quad(51.7)^{\mathrm{a}}$ & $1,885 \quad(48.0)^{\mathrm{a}}$ & $472(46.9)$ & $1,609 \quad(47.2)$ & $199 \quad(54.1)$ & $620 \quad(51.4)$ \\
\hline Female & $492(48.3)^{\mathrm{a}}$ & $2,043(52.0)^{\mathrm{a}}$ & $534 \quad(53.1)$ & $1,800 \quad(52.8)$ & $169 \quad(45.9)$ & $587 \quad(48.6)$ \\
\hline Mean age [SD] & $79.9 \quad[ \pm 9.8]^{\mathrm{b}}$ & $80.5 \quad[ \pm 9.6]^{\mathrm{b}}$ & $80.5[ \pm 10.4]$ & $80.8 \quad[ \pm 9.9]$ & $79.2[ \pm 8.5]$ & $79.9 \quad[ \pm 8.9]$ \\
\hline Mean chronic medications [SD] & $8.5 \quad[ \pm 5.2]^{\mathrm{c}}$ & $7.8 \quad[ \pm 5.6]^{\mathrm{c}}$ & $9.6[ \pm 5.1]^{\mathrm{c}}$ & $9.1 \quad[ \pm 5.5]^{\mathrm{c}}$ & $11.2[ \pm 4.8]^{\mathrm{b}}$ & $10.8[ \pm 5.7]^{\mathrm{b}}$ \\
\hline Proportion of adherent patients, n (\%) & $851 \quad(83.6)^{\mathrm{d}}$ & $3,001 \quad(76.4)^{\mathrm{d}}$ & $777 \quad(77.5)^{\mathrm{a}}$ & $2,509 \quad(73.6)^{\mathrm{a}}$ & $305(82.9)^{\mathrm{a}}$ & $919(76.1)^{\mathrm{a}}$ \\
\hline \multicolumn{7}{|c|}{$\begin{array}{l}{ }^{a} \text { Chi-square test within each adherence metric, } 1 \mathrm{df}, P<0.05 \text {. } \\
{ }^{b} \text { Mann-Whitney } U \text { between study cohorts, } P<0.05 \text {. } \\
{ }^{c} \text { Mann-Whitney } U \text { between study cohorts, } P<0.001 \text {. } \\
{ }^{d} \text { Chi-square test within each adherence metric, } 1 \mathrm{df}, P<0.001 \text {. } \\
d f=\text { degrees of freedom; RASA = renin angiotensin aldosterone sys }\end{array}$} \\
\hline
\end{tabular}

Many research studies have demonstrated the ability of pharmacists to improve medication adherence..$^{3-6}$ Results of community pharmacist interventions designed to improve patient medication adherence show that pharmacists may indirectly affect (i.e., increase) CMS star rating adherence metrics. ${ }^{7}$ A type of intervention that has been adopted extensively by community pharmacies and successfully integrated into practice is automatic prescription refill programs. ${ }^{8}$

Automatic prescription refill programs initiate prescription refills on a standardized, recurrent basis up to 1 week before a patient runs out of medication. The automatic refill program removes the need for patients to stop in or telephone prescription refills to the pharmacy. Automatic refill programs are viewed conceptually as a method for improving patient medication adherence and subsequently CMS star ratings, ${ }^{9}$ but no previous research has confirmed these lay literature reports. The purpose of this study was to compare patient adherence rates, using 3 CMS metrics, for patients enrolled in an automatic prescription refill program with those from patients using the standard refill process.

\section{Methods}

A retrospective, post-only, quasi-experimental design was used for this study. Patients were grouped into 2 cohorts based on whether they were enrolled in an automatic prescription refill program or used the standard refill process. Patients were enrolled in the automatic refill program at all pharmacy locations. Patients taking chronic medications were recruited by the pharmacy staff and were asked to enroll individual medications into the program. The next refill was generated by the dispensing software based on the date the patient last picked up medication. For example, a 30-day supply refill was filled on the twenty-third day after the previous fill was picked up. The study period was January 1, 2014-December 31, 2014. This study was approved by the institutional review board at the authors' institution.

\section{Data Source}

Prescription dispensing data were obtained from a 29-store pharmacy chain in a midwestern state. The majority of the pharmacies in the chain are located in rural suburbs. The demographics of patients in each study cohort are presented in Table 1. A prescription dispensing database containing information for all prescriptions processed by the pharmacy chain from the calendar year 2014 was obtained. Variables included in the claims database were patient birth date, gender, National Drug Code (NDC) number of the drug dispensed, prescription fill date, days supply, quantity dispensed, directions for use, drug name, and automatic refill program indicator (yes/no). Although a prescription insurance identifier was present in the dispensing database, it was not possible to identify Medicare Advantage Plans.

\section{Patients}

Patients included in this study were aged 65 years or older on or before January 1, 2014, and were taking at least 1 medication in the therapeutic categories used in calculating the PDC measures included in this study. The medication adherence metric for lipid therapy (statin metric) includes only HMG-CoA reductase inhibitors (i.e., statins). The medication adherence metric for hypertension therapy (RASA metric) includes only angiotensin-converting enzyme inhibitors and angiotensin-II receptor antagonists. The medication adherence metric for noninsulin diabetes therapy (diabetes metric) includes sulfonylureas, biguanides, dipeptidyl peptidase IV inhibitors, thiazolidinediones, and subtype II sodium-glucose transport protein inhibitors. Patients who had the same medication filled as an automatic refill and a standard refill were not included in the analysis. This exclusion was made in order to isolate the effect of being in only 1 of the 2 refill types. No other adherence programs were being conducted concurrently. Patients must have had at least 2 prescription refills of the included 


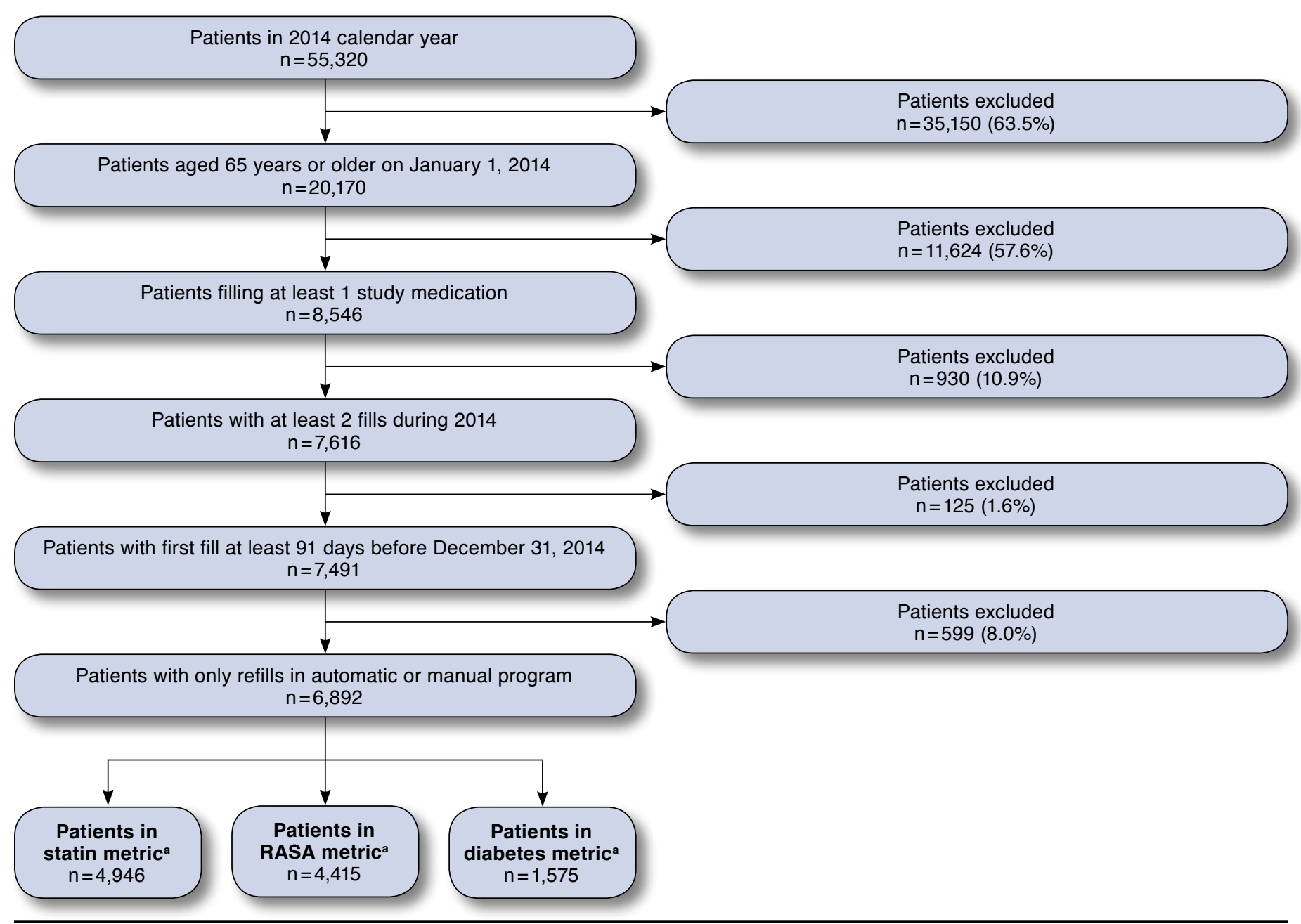

aSome patients were included in more than 1 adherence metric cohort.

RASA = renin angiotensin aldosterone system antagonists.

medications during the 2014 calendar year. The first fill for included medications had to occur at least 91 days before the end of the study period (December 31, 2014). This criterion ensured that patients receiving 90-day supplies of medication were able to obtain a second fill during the study period. The study inclusion criteria were consistent with CMS methodology for calculating adherence for the Part D star rating program. ${ }^{2}$ Figure 1 shows the sample selection flowchart for the number of patients included in each of the 3 medication adherence metrics considered in this study based on the inclusion criteria.

Proportion of days covered (PDC) was used to calculate medication refill adherence. PDC was initially proposed in 2002 as a new method to calculate medication refill adherence using prescription claims data. ${ }^{10} \mathrm{PDC}$ is considered to be an improvement over medication possession ratio because it accounts for medication persistence. As a result, PDC is less likely to bias adherence calculations upward when considering patients who have discontinued their medications. Although PDC is more consistently defined when compared with medication possession ratio, variations of the PDC calculation still exist. ${ }^{7}$ One of these variations includes whether or not to measure concurrent medication use for patients taking more than 1 medication for a single condition (e.g., diabetes). ${ }^{11} \mathrm{CMS}$ uses a PDC calculation developed by the PQA., ${ }^{2,12}$ This PDC calculation is based on the prescription fill date and days supply for each fill of a prescription. Consistent with the PQA calculation, the fill date in this study was defined as the date that the prescription claim was submitted to the pharmacy benefits 
manager for payment. Days supply was defined as the number of days the medication fill would last based on the written directions on the prescription and was represented by the days supply value entered by an employee of the pharmacy. The PDC calculation used in this study is as follows:

$$
P D C=\frac{\text { Number of days covered with medication }}{\text { Number of days in observation period }}
$$

The numerator is the number of days that a patient had medication available to take based on the prescription fill dates and days supplies. The denominator is the number of days between the last day in the calendar year (December 31, 2014) and the date of the patient's first fill of a medication included in the adherence metric during 2014. To account for the possibility that a patient used multiple NDC numbers for the same medication within an adherence metric (e.g., multiple strengths of the same medication), a Generic Product Identifier available from PQA was used. The Generic Product Identifier aggregated NDC numberss to the generic drug name level. Additionally, the PDC calculation accounted for overlapping days supply (e.g., a refill is obtained before the quantity of medication from the previous fill is gone) by adjusting the start date of the next fill to be equal to the previous fill plus the days supply. This adjustment also occurred when patients switched between 2 NDC numbers of the same medication. Situations in which patients used more than 1 medication in a single adherence metric (e.g., multiple diabetes medications) or had a dose change during the study period did not result in an adjustment to the fill date, since the patient likely took the medications concurrently or stopped one in favor of the other. To be considered a covered day, patients who used more than 1 medication considered in the adherence metric only needed to have 1 of the medications available to them based on the prescription fill date.

\section{Variables}

A dichotomous variable (adherent $=1$, not adherent $=0$ ) was created using an 80\% PDC criterion for automatic and standard refill prescriptions for each of the 3 adherence metrics. This is the same criterion recommended by the PQA and used by CMS to decide whether patients are adherent to their medications. ${ }^{2}$ The proportion of patients in each study cohort adherent to their medications was calculated using the following formula:

Proportion of patients with $\mathrm{PDC}>80 \%=$ Number of patients with $\mathrm{PDC}>80 \%$

$$
\text { Total number of patients }
$$

Independent variables used in the analysis included age, gender, automatic prescription refill program status, and the number of chronic medications used by the patient. The number of chronic medications served as a proxy for a patient's health status. Birth dates were converted to age by subtracting the patient's date of birth from January 1, 2014, to give age at the beginning of 2014. Chronic medications were defined as those medications with at least 2 fills during the 2014 calendar year, with the first fill occurring at least 91 days before December 31, 2014. To determine the number of chronic medications, the number of unique NDC numbers dispensed to each patient was determined. A Generic Product Identifier was not available for all medications dispensed to a patient, so it is possible that the calculated number of NDC numbers overestimated the number of chronic medications, since patients could switch between 2 NDC numbers of the same generic medication. If this occurred, each NDC number was counted as a chronic medication.

\section{Data Analysis}

The chi-square statistic was used to test for differences in the proportion of adherent patients in the automatic refill program or the standard refill process. Two assumptions for performing a chi-square test were (1) the independence of observations and (2) a minimum expected frequency of observations in each cell of the contingency table. Both of these assumptions were met with the data.

Pending a significant chi-square test, logistic regression analysis was used to examine the association of each independent variable on whether a person was adherent. ${ }^{13}$ To determine the usefulness of performing multilevel modeling, the intraclass correlation coefficient was calculated. ${ }^{14,15}$ The intraclass correlation coefficients for each adherence metric were all close to zero. Diagnostics of assumptions to confirm the use of logistic regression were performed. Bivariate logistic regression was performed first, then multivariate regression was used to examine the relationships while controlling for the other variables in the analysis. Only the multivariate logistic regression results are presented.

All data cleaning, preprocessing, and statistical analysis were performed using R3.2.2 (R Foundation for Statistical Computing, Vienna, Austria).

\section{Results}

Table 1 provides summary descriptive characteristics for patients in each study cohort across the 3 adherence metric categories. The percentage of patients enrolled in the automatic prescription refill program was $20.5 \%, 22.8 \%$, and $23.3 \%$ in the statin, RASA, and diabetes metrics, respectively. For the statin adherence metric, there were a significantly greater proportion of males in the automatic refill program cohort. There were statistically significant differences in the age of patients in the automatic refill program compared with the standard refill process for the statin metric, but not in the RASA or diabetes metrics. For the statin adherence metric, there was a significantly greater proportion of males in the automatic refill program cohort. Across each adherence metric category, patients in the automatic refill cohort had less than 1 additional chronic medication compared with the standard refill 
TABLE 2 Multivariate Adjusted Odds Ratios of Being Adherent by Adherence Metric

\begin{tabular}{|c|c|c|c|c|c|c|}
\hline & \multicolumn{2}{|c|}{ Statin } & \multicolumn{2}{|c|}{ RASA } & \multicolumn{2}{|c|}{ Diabetes } \\
\hline & $\begin{array}{c}\text { Adjusted Odds } \\
\text { Ratio } \\
\end{array}$ & $95 \%$ CI & $\begin{array}{c}\text { Adjusted Odds } \\
\text { Ratio } \\
\end{array}$ & $95 \%$ CI & $\begin{array}{c}\text { Adjusted Odds } \\
\text { Ratio } \\
\end{array}$ & $95 \% \mathrm{CI}$ \\
\hline \multicolumn{7}{|l|}{ Refill type } \\
\hline Automatic fill & $1.51^{\mathrm{a}}$ & $1.26-1.82$ & $1.20^{\mathrm{b}}$ & $1.01-1.42$ & $1.44^{b}$ & $1.06-1.95$ \\
\hline \multicolumn{7}{|l|}{ Gender } \\
\hline Male & 1.09 & $0.95-1.25$ & 1.04 & $0.91-1.20$ & 0.98 & $0.77-1.25$ \\
\hline Age & 0.99 & $0.99-1.00$ & 0.99 & $0.98-1.01$ & 0.98 & $0.97-1.00$ \\
\hline Number of chronic medications & $1.03^{\mathrm{a}}$ & $1.02-1.04$ & 1.00 & $0.99-1.01$ & $1.05^{\mathrm{a}}$ & $1.02-1.07$ \\
\hline \multicolumn{7}{|c|}{$\begin{array}{l}\text { Note: Standard refill and female were reference categories. } \\
a_{P}<0.001 . \\
b P<0.05 \text {. } \\
C I=\text { confidence interval; RASA = renin angiotensin aldosterone system antagonists. }\end{array}$} \\
\hline
\end{tabular}

cohort. The difference in number of chronic medications was statistically significant across all adherence metrics. For each adherence metric category, the percentage of patients who were adherent was significantly higher in the automatic refill cohort compared with the standard refill group cohort

The output of the odds ratios from the multivariate logistic regression models for each adherence category are presented in Table 2. Patients in the automatic refill program were significantly more likely to be adherent to their medications relative to the standard refill cohort for each of the adherence metrics when controlling for age and gender. For the statin adherence metric, patients in the automatic refill program were more likely to be adherent to their medications, controlling for the other variables (odds ratio $[O R]=1.51,95 \%$ confidence interval $[C I]=1.26-1.82$ ). No significant increases in the likelihood of being adherent to statins were seen for age or gender. For each additional chronic medication, patients in the statin cohort were more likely to be adherent when controlling for the other variables $(\mathrm{OR}=1.03,95 \% \mathrm{CI}=1.02-1.04)$.

For the RASA medication adherence metric, patients in the automatic refill cohort were more likely to be adherent to their medications compared with standard refills $(\mathrm{OR}=1.20$, 95\% CI=1.01-1.42). None of the other independent variables were found to be associated with an increased likelihood of being adherent. Patients in the diabetes adherence metric were more likely to be adherent when comparing the automatic prescription refill cohort to the standard refill cohort $(\mathrm{OR}=1.44$, $95 \% \mathrm{CI}=1.06-1.95)$. No significant ORs were found for age or gender, but for each additional chronic medication, patients were more likely to be adherent to their diabetes medications $(\mathrm{OR}=1.05,95 \% \mathrm{CI}=1.02-1.07)$.

\section{Discussion}

The purpose of this study was to compare the proportion of patients considered adherent to their medications who were enrolled in an automatic refill program compared with patients refilling prescriptions using the standard, nonautomatic method. There is a dearth of published evidence on whether adherence rates are improved when patients are enrolled in automatic refill programs for prescription medications relative to patients using the standard refill process. The differences in the proportion of patients considered adherent to their medications were $7.2 \%, 3.9 \%$, and $6.8 \%$ higher in the automatic prescription refill cohort for statin, RASA, and diabetes metrics, respectively. The odds of patients enrolled in an automatic prescription refill program being adherent were higher compared with the standard refill cohort for each of the adherence metrics. These results may have significant implications for CMS, health plans, and pharmacies.

A main goal for CMS is the improvement of health outcomes for their beneficiaries. Increasing adherence to medication therapy has been shown to improve outcomes through improved quality of life and decreased morbidity and mortality, ${ }^{7,16}$ The increase in the proportion of adherent patients resulting from an automatic prescription refill program compared with the standard refill process may help CMS to achieve this goal. At the same time, it is not clear whether improvements in PDC rates are divergent from actual improvements in patient ingestion of the medications. For patients who pick up their automatically refilled prescriptions from a retail pharmacy, it is generally assumed that they consume the medications. Larger concerns, however, have been expressed over automatic shipment programs from mail-order pharmacies, in which the patient does not actively pick up the medication. ${ }^{8,17}$

One of the purposes of the Medicare star ratings is to support improvement in health outcomes for patients. Additionally, the star ratings are publicly reported and can be viewed by Medicare beneficiaries who are considering enrollment in Medicare plans. CMS provides bonus payments of $5 \%$ per enrollee to Medicare Advantage Plans that are able to achieve a 4- or 5-star overall rating. This can translate into significant additional revenue for these health plans. In 2012, it was 
estimated that CMS paid $\$ 281$ in bonus payments per enrollee, or a total of $\$ 3.1$ billion, to Medicare Advantage Plans. ${ }^{18}$ Medicare Advantage and stand-alone Medicare Prescription Drug Plans also receive a star rating for each of the 3 adherence metrics examined in this study. As a result, health plans have multiple incentives for increasing the proportion of patients that are adherent to their medications. The results of this study suggest that encouraging patient enrollment in an automatic prescription refill program is a way to achieve this objective.

Pharmacies and pharmacists may benefit in many ways from increasing patient enrollment in automatic prescription refill programs. First, automatic refill programs may help patients achieve better health if improved refill adherence translates to patients taking their medications as directed. Second, if patients take their medications as directed, they likely will purchase more medications, resulting in more revenue for the pharmacy. The additional revenue can improve the financial health of a pharmacy. Additionally, pharmacy revenue could increase if health plans contract only with pharmacies that have higher refill adherence rates. Certain health plans have also elected to provide bonus payments to a pharmacy with a higher proportion of adherent patients..$^{19}$ In the future, pharmacies that have a high proportion of patients who are adherent to their medications may receive a portion of the bonus payments that health plans receive from CMS. ${ }^{18}$

\section{Limitations}

This research has several limitations. First, questions about the validity of using pharmacy dispensing or administrative claims data to measure adherence have been raised. ${ }^{20,21}$ One of the major assumptions of using these data is that refill adherence equals adherence with medication ingestion. Previous research suggests that for the same patient and medication, variable rates of adherence can occur depending on the method used (e.g., pill counts, self-reporting, and claims data). ${ }^{20,21}$ However, use of dispensing and claims data is one of the only methods for estimating adherence on a large scale and is used extensively by researchers.

Second, other important limitations were the lack of random assignment to the automatic prescription refill program and the unavailability of detailed patient demographic information to the researchers. Additional variables could have been used in a propensity score matching approach to reduce threats to validity caused by nonrandom assignment to the refill programs. Lack of random assignment to each cohort limits the conclusions that can be drawn about the true effect that the automatic prescription refill program has on medication refill adherence. While an association between automatic prescription refills and PDC was found, it is not possible to conclude that a causal relationship exists. ${ }^{22}$ In addition, the findings may not be generalizable to other pharmacy populations and other automatic prescription refill programs.
Finally, the patient data used for this analysis were collected from pharmacies, not health plans. Pharmacy patient data do not allow researchers to include prescriptions refilled outside of the pharmacy chain, whereas health plan patient data would include prescription refills from multiple different pharmacy organizations.

\section{Conclusions}

An automatic prescription refill program was associated with a higher proportion of patients that were considered adherent to their medications compared with the standard refill process. The results suggest that patients could be encouraged by pharmacists to enroll in automatic prescription refill programs in order to improve Medicare Part D star ratings from CMS. However, the extent to which automatic prescription refill programs improve the rate at which patients actually take their medications is unknown, since prescription dispensing data only measure the number of days a patient would have medication available to take. Future research should address this concern.

\section{Authors}

COREY A. LESTER, PharmD, MS; DAVID A. MOTT, PhD; and MICHELLE A. CHUI, PharmD, PhD, Social and Administrative Sciences Division, School of Pharmacy, University of WisconsinMadison.

AUTHOR CORRESPONDENCE: Corey A. Lester, PharmD, MS, School of Pharmacy, University of Wisconsin-Madison, 777 Highland Ave., Madison, WI 53705. Tel.: 608.262.0452; Fax: 608.262.5262; E-mail:calester@wisc.edu.

\section{DISCLOSURES}

The project described was supported by the Clinical and Translational Science Award (CTSA) program through the NIH National Center for Advancing Translational Sciences (NCATS), grant ULITR000427. The content is solely the responsibility of the authors and does not necessarily represent the official views of the NIH. Lester is employed as a pharmacist in the participating pharmacy chain. The authors report no other relevant conflict of interest.

Study concept and design were primarily contributed by Lester, with assistance from the other authors. Lester took the lead in data collection, along with Chui, and data interpretation was performed by Lester, Mott, and Chui. The manuscript was written primarily by Lester, along with Mott, and revised by Lester, Mott, and Chui.

\section{REFERENCES}

1. Pharmacy Quality Alliance. About PQA. Available at: http://pqaalliance. org/about/default.asp. Accessed May 9, 2016.

2. Centers for Medicare \& Medicaid. Medicare 2016 Part C \& D star rating technical notes: first plan preview. Draft. August 5, 2015. Available at: https://www.cms.gov/Medicare/Prescription-Drug-Coverage/

PrescriptionDrugCovGenIn/Downloads/2016-Technical-Notes-Preview1-v2015_08_05.pdf. Accessed May 9, 2016. 
3. Ratanawongsa N, Karter AJ, Parker MM, et al. Communication and medication refill adherence: the Diabetes Study of Northern California. JAMA Intern Med. 2013;173(3):210-18.

4. Twigg MJ, Bhattacharya D, Desborough JA, Wright D. A drop-in clinic for patients with poorly-controlled diabetes: a community pharmacy feasibility study. Int J Clin Pharm. 2015;37(2):395-402.

5. Holdford DA, Inocencio TJ. Adherence and persistence associated with an appointment-based medication synchronization program. J Am Pharm Assoc. 2013;53(6):576-83

6. Pringle JL, Boyer A, Conklin MH, McCullough JW, Aldridge A. The Pennsylvania Project: pharmacist intervention improved medication adherence and reduced health care costs. Health Aff (Millwood). 2014;33(8):1444-452.

7. Lee JK, Grace KA, Taylor AJ. Effect of a pharmacy care program on medication adherence and persistence, blood pressure, and low-density lipoprotein cholesterol: a randomized controlled trial. JAMA. 2006;296(21):2563-71.

8. Morran C. Pharmacists confirm pressure from management to refill prescriptions automatically. Consumerist. October 25, 2012. Available at: http:// consumerist.com/2012/10/25/pharmacists-confirm-pressure-from-management-to-refill-prescriptions-automatically/. Accessed May 9, 2016.

9. Blank C. PPACA moves medication adherence to center stage. Drug Topics. April 15, 2011. Available at: http://drugtopics.modernmedicine.com/ node/149483. Accessed May 9, 2016.

10. Benner JS, Glynn RJ, Mogun H, Neumann PJ, Weinstein MC, Avorn J. Long-term persistence in use of statin therapy in elderly patients. JAMA. 2002;288(4):455-61.

11. Choudhry NK, Shrank WH, Levin RL, et al. Measuring concurrent adherence to multiple related medications. Am J Manag Care. 2009; 15(7):457-64.

12. Nau DP. Proportion of days covered (PDC) as a preferred method of measuring medication adherence. Pharmacy Quality Alliance. 2012. Available at: http://ep.yimg.com/ty/cdn/epill/pdcmpr.pdf. Accessed May 9, 2016.
13. Bewick V, Cheek L, Ball J. Statistics review 14: logistic regression. Crit Care. 2005;9(1):112-18.

14. Cress U. The need for considering multilevel analysis in CSCL research-an appeal for the use of more advanced statistical methods. International Journal of Computer-Supported Collaborative Learning. 2008;3(1):69-84.

15. Donner A, Wells G. A comparison of confidence interval methods for the intraclass correlation coefficient. Biometrics. 1986;42(2):401-12.

16. Bramley TJ, Gerbino PP, Nightengale BS, Frech-Tamas F. Relationship of blood pressure control to adherence with antihypertensive monotherapy in 13 managed care organizations. J Manag Care Pharm. 2006;12(3):239-45. Available at: http://amcp.org/data/jmcp/Research_239-245.pdf.

17. Robinson S. Community pharmacy and CMS star ratings: how technology can increase efficiency and quality of care. Drug Topics. August 10, 2014. Available at: http://drugtopics.modernmedicine.com/drug-topics/content/ tags/cms-star-ratings/community-pharmacy-and-cms-star-ratings?page=full. Accessed May 9, 2016.

18. Dharmarajan S, Bentley JP, Banahan BF III, West-Strum DS. Measuring pharmacy performance in the area of medication adherence: addressing the issue of risk adjustment. J Manag Care Pharm. 2014;20(10):1057-68. Available at: http://www.jmcp.org/doi/abs/10.18553/jmcp.2014.20.10.1057.

19. American Pharmacists Association. Health plan starts P4P program for community pharmacies. February 1, 2014. Available at: https://www. pharmacist.com/health-plan-starts-p4p-program-community-pharmacies. Accessed May 9, 2016.

20. Garber MC, Nau DP, Erickson SR, Aikens JE, Lawrence JB. The concordance of self-report with other measures of medication adherence: a summary of the literature. Med Care. 2004;42(7):649-52.

21. Gao X, Nau DP. Congruence of three self-report measures of medication adherence among HIV patients. Ann Pharmacother. 2000;34(10):1117-22.

22. Nichols A. Causal inference with observational data. Stata J. 2007;7(4):507. 